\title{
Variability in the light curve of tidal disruption events $\dagger$
}

\author{
Zu-Jia Lu ${ }^{1,2}$ ，Da-Bin Lin ${ }^{1,2}$, Ling-Hua Xie ${ }^{1,2}$ and En-Wei Liang ${ }^{1,2}$ \\ ${ }^{1}$ GXU-NAOC Center for Astrophysics and Space Sciences, Department of Physics, \\ Guangxi University, Nanning 530004, China, email: lindabin@gxu.edu.cn; lew@gxu.edu.cn \\ ${ }^{2}$ Guangxi Key Laboratory for Relativistic Astrophysics, Nanning 530004, China
}

The X-ray light curve of Sw J1644+57 indicates this event would be due to a tidal disruption. The lightcurve shows large amplitude fluctuation. As proposed by Lyubarskii (1997), the aperiodic variability observed in the Galactic X-ray binaries and active galactic nuclei is likely from the fluctuation of the viscous parameter in their disks. We explain the significant fluctuation of the late X-ray lightcurve $\left(t>10^{6}\right.$ seconds) of Sw J1644+57 with this model. We assume the stochastic variations in the viscous parameter featuring as $\alpha(R, t)=\alpha_{0}[1+\beta(R, t)]$, where the time-scale for varying $\beta(R, t)$ is set as ten times of the dynamic time-scale for disk at the radius $R$ (Janiuk \& Misra 2012). Based on the simulation results of Lodato et al. (2009), we describe the fallback behavior of the tidal disruption as $\dot{M}_{\mathrm{fb}} \propto\left\{\left[\left(t-t_{b}\right) / t_{f b}\right]^{\kappa n}+\left[\left(t-t_{b}\right) / t_{f b}\right]^{5 n / 3}\right\}^{-1 / n}$ for $t>t_{b}$ and $\dot{M}_{\mathrm{fb}}=0$ for other situations, where $\kappa=10.0, n=0.5, t_{\mathrm{fb}}=10^{3} \tau$, and $t_{b}=10^{2} \tau$ in which $\tau=2 \pi\left(R_{f}^{3} / G M_{\mathrm{BH}}\right)^{1 / 2}$ and $R_{f}=5 r_{g}$ is the pericentre distance. Figure 1 compare the power-density spectra (PDS) derived from the observed and our simulated lightcurves. It is found the our simulations are well consistent with the observations.

Keywords. accretion, accretion disks - black hole physics - galaxies: nuclei - X-rays: binaries
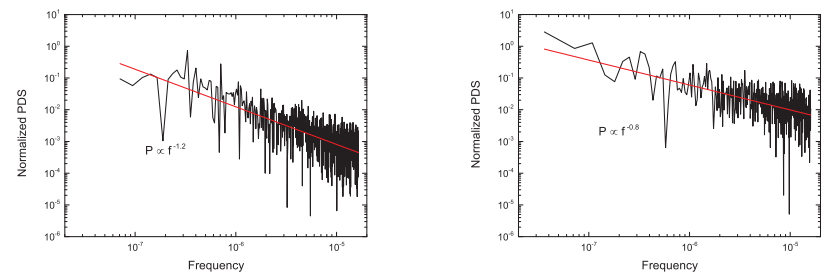

Figure 1. Comparison the power spectrum density of the observed (left) and our simulated lightcurves (right).

\section{References}

Janiuk, A., \& Misra, R., 2012, A\&3A, 540, A114

Lyubarskii, Y. E., 1997, MNRAS, 292, 679

Lodato, G., King, A. R., Pringle, J. E., 2009, MNRAS, 332, 340

$\dagger$ Supported by the National Basic Research Program (973 Programme) of China (Grant No. 2014CB845800), the National Natural Science Foundation of China (Grant Nos. 11533003, 11403005), the Guangxi Science Foundation (2013GXNSFFA019001 and 2014GXNSFBA118004), the Strategic Priority Research Program "The Emergence of Cosmological Structures"(Grant No. XDB09000000). 